\title{
Microstructure and Interfaces of Ultra-Thin Epitaxial AlN Films Grown by Plasma-Enhanced Atomic Layer Deposition at Relatively Low Temperatures
}

\author{
Ramasis Goswami ${ }^{1, *}$, Syed Qadri ${ }^{1}$, Neeraj Nepal ${ }^{2}$ and Charles Eddy, Jr. ${ }^{2}$ \\ 1 Naval Research Laboratory, Materials Science and Technology Division, 4555 Overlook Ave. SW, \\ Washington, DC 20375, USA; syed.qadri@nrl.navy.mil \\ 2 Naval Research Laboratory, Electronics Science \& Technology Division, 4555 Overlook Ave. SW, \\ Washington, DC 20375, USA; neeraj.nepal@nrl.navy.mil (N.N.); charles.eddy@nrl.navy.mil (C.E.J.) \\ * Correspondence: ramasis.goswami@nrl.navy.mil
}

check for updates

Citation: Goswami, R.; Qadri, S.; Nepal, N.; Eddy, C., Jr. Microstructure and Interfaces of Ultra-Thin Epitaxial AlN Films Grown by

Plasma-Enhanced Atomic Layer Deposition at Relatively Low Temperatures. Coatings 2021, 11, 482. https://doi.org/10.3390/ coatings11040482

Academic Editor: Alessio Lamperti

Received: 16 March 2021

Accepted: 7 April 2021

Published: 20 April 2021

Publisher's Note: MDPI stays neutral with regard to jurisdictional claims in published maps and institutional affiliations.

Copyright: (c) 2021 by the authors. Licensee MDPI, Basel, Switzerland. This article is an open access article distributed under the terms and conditions of the Creative Commons Attribution (CC BY) license (https:// creativecommons.org/licenses/by/ $4.0 /)$.

\begin{abstract}
We demonstrate the growth of ultra-thin AlN films on Si (111) and on a GaN/sapphire (0001) substrate using atomic layer epitaxy in the temperature range of 360 to $420{ }^{\circ} \mathrm{C}$. Transmission electron microscopy and X-ray diffraction were used to characterize the interfaces, fine scale microstructure, and the crystalline quality of thin films. Films were deposited epitaxily on Si (111) with a hexagonal structure, while on the GaN/sapphire (0001) substrate, the AlN film is epitaxial and has been deposited in a metastable zinc-blende cubic phase. Transmission electron microscopy reveals that the interface is not sharp, containing an intermixing layer with cubic AIN. We show that the substrate, particularly the strain, plays a major role in dictating the crystal structure of AlN. The strain, estimated in the observed orientation relation, is significantly lower for cubic AIN on hexagonal GaN as compared to the hexagonal AlN on hexagonal GaN. On the Si (111) substrate, on the other hand, the strain in the observed orientation relation is $0.8 \%$ for hexagonal AlN, which is substantially lower than the strain estimated for the cubic $\mathrm{AlN}$ on $\mathrm{Si}(111)$.
\end{abstract}

Keywords: atomic layer deposition; aluminum nitride; gallium nitride; interfaces; transmission electron microscopy

\section{Introduction}

Aluminum nitride is a III-N compound semiconductor with a band gap in the range of 6.01-6.05 eV at room temperature [1]. This semiconducting material is a useful candidate in a number of potential applications in microelectronics because of its relatively high thermal conductivity, high electrical resistivity, low thermal expansion, chemical stability in air up to $1380{ }^{\circ} \mathrm{C}$, and excellent thermal shock resistance. The epitaxial crystalline aluminum nitrides are used to manufacture surface acoustic wave sensors because of the piezoelectric properties of AlN [2,3]. It exhibits both wurtzite and zinc-blende phases as do other III-N compounds. In the present paper, we report on the low-temperature growth of ultra-thin $(<20 \mathrm{~nm})$ binary AlN films using atomic layer deposition (ALD) growth processes. The low-temperature growth process allows the elimination of miscibility gaps in ternary III-N semiconductors and reduces extended defect formation due to thermal coefficient of expansion mismatch that affects the quality of the films grown with the conventional higher temperature growth methods. Metal-organic chemical vapor deposition (MOCVD) and molecular beam epitaxy have been used to grow good quality AlN films. However, it involves high temperatures that results in the obstruction of growing strain-free heteroepitaxial film as well as the full stoichiometric range of alloyed III nitrides [2-5]. The miscibility gap in AIN occurs above $500{ }^{\circ} \mathrm{C}$, which can lead to spinodal decomposition during growth at these temperatures. At the present time, the III-nitride films of highest quality are deposited by molecular beam epitaxy and metalorganic chemical vapor depo- 
sition methods. It has been reported that temperatures of $900{ }^{\circ} \mathrm{C}$ and higher are usually needed to obtain high-quality AlN films by these methods.

The epitaxial growth of AlN has been carried out at high temperatures, usually at temperatures greater than $1300{ }^{\circ} \mathrm{C}$, which enables the bulk diffusion of $\mathrm{Al}$ and reduces the density of defects $[6,7]$. The growth involves different methods at a relatively high temperature, such as epitaxial lateral overgrowth [8-11], pulsed atomic layer epitaxy [12-16], and high-temperature post-growth annealing [17-19]. However, the high-temperature growth has inherent difficulties as it introduces high thermal stresses and buckling due to the thermal expansion mismatch between the substrate and AlN film [20].

To overcome these difficulties, currently, the ALD technique has been largely explored to deposit AlN films at relatively low temperature of $640^{\circ} \mathrm{C}$ [21] as the dimensions of the device are reduced and the non-planar complexity is enhanced [22]. However, films grown at reduced temperatures, $1100-1200{ }^{\circ} \mathrm{C}$, contain a high density of point defects [23,24] and threading dislocations [25-28]. These films also exhibit rough surface morphology [26,28]. In addition, the low-temperature ALD growth could yield amorphous film or metastable phases in the film. Recently, ALD growth for AlN has been reported in the temperature range of $150-280^{\circ} \mathrm{C}$ using tris (diethylamido) aluminum and hydrazine (N2H4) or ammonia [29] and in the temperature range of $175-350^{\circ} \mathrm{C}$ using trimethyl aluminum (TMA) and hydrazine [30]. The use of highly reactive nitrogen source, such as hydrazine, provides a higher deposition rate per cycle as compared to ammonia. However, the low-temperature deposition of AlN shows a higher level of impurity content, particularly hydrogen [29].

To facilitate epitaxial films at relatively low temperatures, plasma-enhanced ALD (PDALD) processes can be used [30,31]. In this process, films are grown layer-by-layer with adsorption of the group III-containing molecule first, followed independently by adsorption/reaction with the group $\mathrm{V}$ atom. This is actually a non-equilibrium growth process that is comprised of two surface-limited half-reactions. Thus, the growth process is a complex one, which uses the metal-organic precursors and reactive radicals from a plasma source. As this process involves surface-mediated chemical reactions, understanding the interface, defects, and chemical composition at the nanoscale and fine-scale microstructure of atomic layer epitaxy grown film would be crucial to control the properties of these films. Not much work has been done to investigate the interfaces and reaction layers at the nanoscale nor the fine-scale microstructure formed as a result of the film deposition. Thus, the objective of this work is to grow ultra-thin AlN films on Si and on GaN/sapphire substrates using PDALD at low temperatures of $360-420{ }^{\circ} \mathrm{C}$ and to investigate the interfaces, defects, and fine-scale microstructure of these films.

\section{Experimental}

\subsection{Growth Parameters}

Initially, the substrates were cleaned using hydrogen plasma; then, they were exposed to nitrogen plasma. The process uses 10-30 pulses of hydrogen and 10 pulses of nitrogen plasma. For AlN deposition, in the first step of the cycle, a trimethyl aluminum (TMA) dose was applied for $0.06 \mathrm{~s}$; then, a $10 \mathrm{~s}$ purge and a $20 \mathrm{~s} 75 \mathrm{sccm}$ nitrogen and $10 \mathrm{sccm}$ hydrogen plasma exposure at $300 \mathrm{~W}$ were applied. Argon was used as a carrier gas for the TMA step. The growth temperatures ranged from 360 to $420{ }^{\circ} \mathrm{C}$, and the growth mode is layer-by-layer growth. A Williamson pyrometer was used to record the temperature before and after the growth. The rate of growth was in the range of 0.7 to $1.0 \AA$ /cycle, and all growths were on $\mathrm{Si}$ and on $\mathrm{GaN} /$ sapphire templates grown by MOCVD.

\subsection{Characterization Techniques: $X R D$ and TEM}

A number of high-resolution X-ray diffraction (XRD) scans of the resulting films were made with $\mathrm{CuK}_{\alpha}$ radiation using a Rigaku $18 \mathrm{~kW}$ rotating anode generator. Transmission electron microscopy (TEM) was employed to investigate the structure and the crystallinity of the ALD layers using a JEOL 2200-FX analytical transmission electron microscope (JEOL Ltd. Tokyo, Japan) operated at an accelerating voltage of $200 \mathrm{kV}$. Cross-sectional TEM 
samples were made by gluing and mechanically polishing two specimens to a thickness of 25-50 $\mu \mathrm{m}$. Finally, the samples were thinned using a Gatan ion-mill, PIPS (Gatan Inc., Pleasanton, CA, USA), operated at a voltage of $4 \mathrm{kV}$ for each gun. Digitalmicrograph ${ }^{\mathrm{TM}}$ software 3.4 was used to obtain the Fast Fourier transforms (FFTs) from the high-resolution transmission electron microscopic (HRTEM) images.

\section{Results and Discussion}

TEM was employed to investigate the structural characteristics of the AlN/Si film and interfaces. Figure 1 a shows the AlN film $\approx 10 \mathrm{~nm}$ thick, which was deposited at $400{ }^{\circ} \mathrm{C}$ on (111) $\mathrm{Si}$ with $\approx 50$ cycles. The film was observed to be deposited as different layers, exhibiting dark and light contrast (see Figure 1b). HRTEM has been conducted to investigate the nature of the films, interfaces, and defects. Figure 2a is the HRTEM image near the [11-2] zone of Si showing that the film is epitaxial on (111) Si. We have not observed any threading dislocations, although it is deposited at relatively low temperature. However, one could observe faults that appeared as wavy morphology in the HRTEM image. The interface between $\mathrm{Si}$ and AlN looks considerably sharp. To analyze the structure of the AlN film, we extracted the fast Fourier transform (FFT) from the film and the substrate (see Figure $2 b$ ), and we compared it with the FFT of the substrate in the [11-2] zone (see the inset of Figure 2a). We observe streaking associated with the reflections from the substrate. The streaking in the 111 direction in the FFT results from the faults in the film. The inverse FFT (IFFT) of the film is shown in Figure 2c. TEM analyses show that the film is hexagonal AlN (SG: $\mathrm{P}_{3} / \mathrm{mmc}(194)$ ), and the d-spacings of $0.15 \mathrm{~nm}$ and $0.19 \mathrm{~nm}$ conform to the spacings of (10-12) and (0002) of hexagonal AlN. Usually, the strain effects in the film are dominant close to the substrate. We have measured the $\mathrm{d}$-spacing from HRTEM $\approx 10 \mathrm{~nm}$ away from the substrate/film interface. One could also observe from TEM images that an orientation relationship exists between the ALD-AIN and $\mathrm{Si}$, which is given by $(111)_{\mathrm{Si}}||(11-20)_{\mathrm{AlN}}$, $(2-20)_{\mathrm{Si}}||(0002)_{\mathrm{AlN}}$ and $[-1-12]_{\mathrm{Si}} \mid$ | [1-100] $]_{\mathrm{AlN}}$.
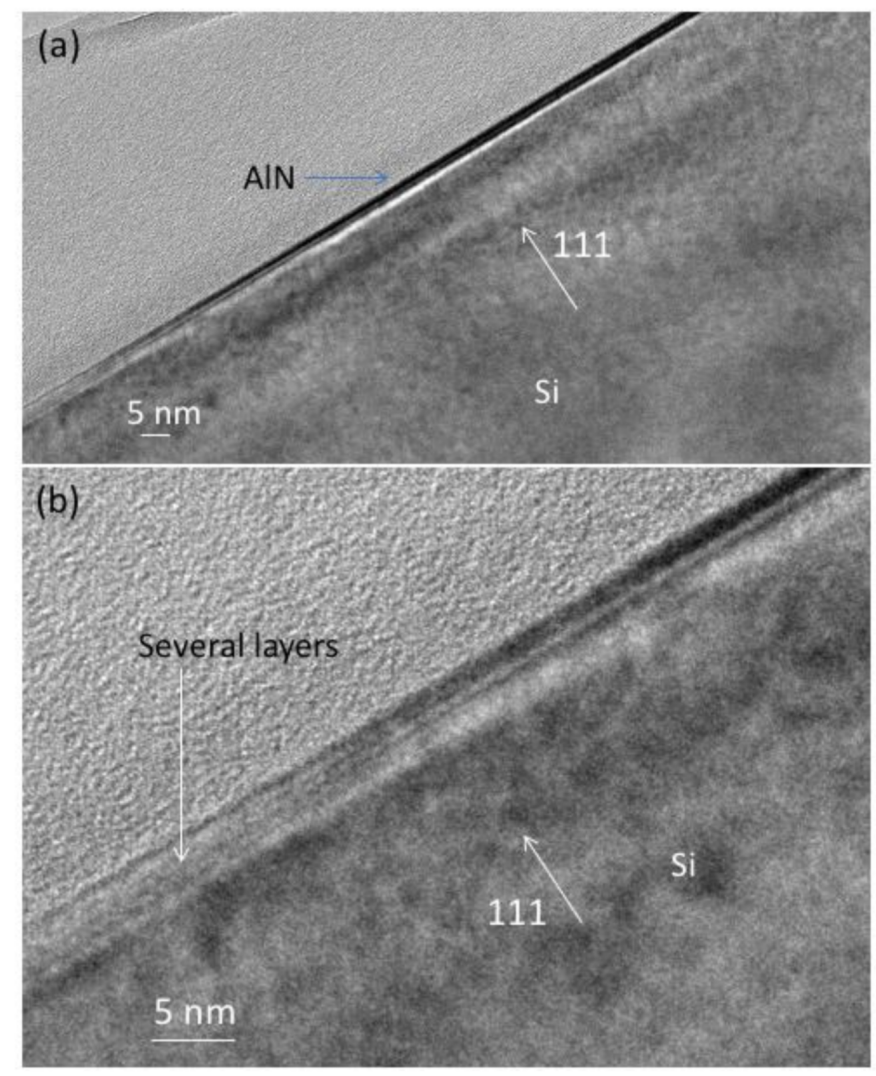

Figure 1. (a) TEM image showing AlN film on $\mathrm{Si}$ (111). (b) A higher magnification multibeam image showing the AlN film consisting of several layers. 

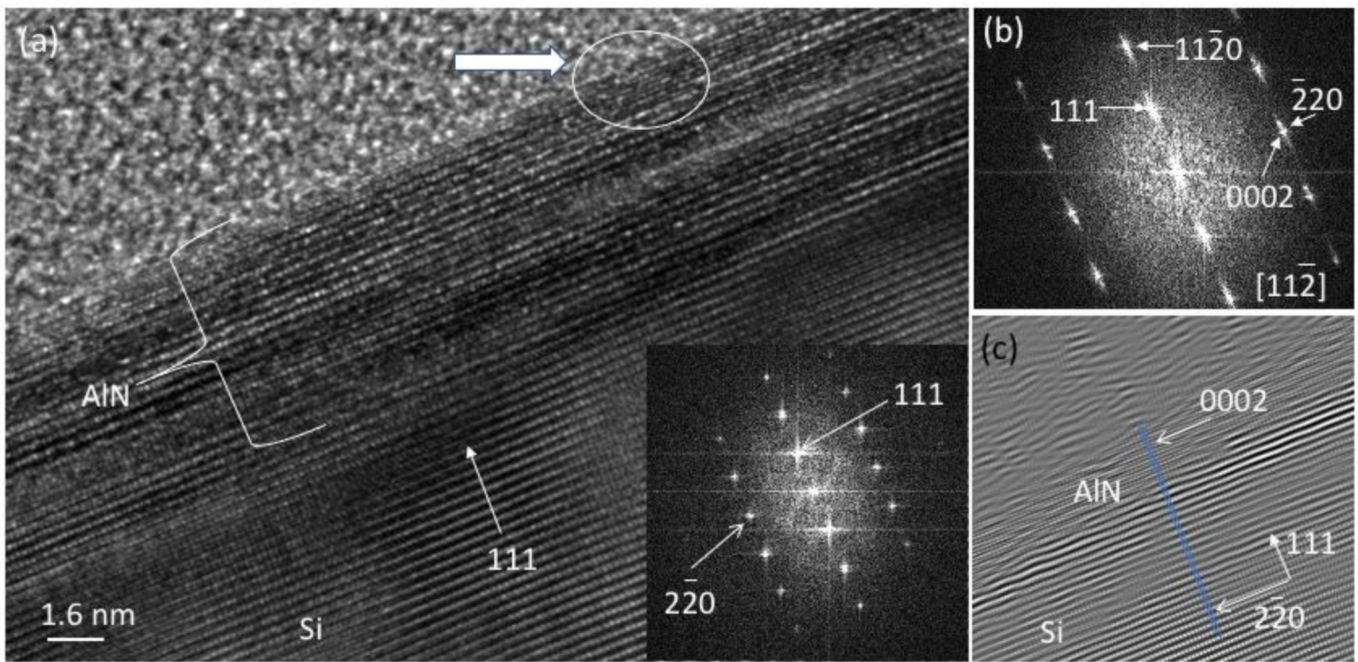

Figure 2. (a) High-resolution transmission electron microscopic (HRTEM) image near the [11-2] zone of Si showing the AlN layer and relative sharper Si/AIN interface. The Fast Fourier transforms (FFT) from the substrate only has been shown as an inset. (b) FFT obtained from the portion of film and the substrate. The spots have been indexed. (c) IFFT image of the AIN film and the substrate.

The HRTEM image near the [112] zone (see Figure 3) of Si from another portion of the film at a higher magnification shows a rather sharp interface with a dot-dashed line. The crystallographic a-direction $\langle 11-20\rangle$ is indicated in the image. In the observed orientation relationship, the estimated strain, $\left(\mathrm{d}_{2-20}{ }^{\mathrm{Si}}-\mathrm{d}_{0002}{ }^{\mathrm{AlN}}\right) / \mathrm{d}_{2-20} \mathrm{Si}$, perpendicular to the growth direction is $0.8 \%$, using $\mathrm{d}_{2-20}{ }^{\mathrm{Si}}=0.191 \mathrm{~nm}$ and $\mathrm{d}_{0002}{ }^{\mathrm{AlN}}=0.1895 \mathrm{~nm}$. The strain type in this case would be tensile. However, for cubic AlN on Si (111), the strain would be more than $10 \%$ as $\mathrm{d}_{200}$ AlN $=0.218 \mathrm{~nm}$, suggesting that the hexagonal AlN would be favored on $\mathrm{Si}$ (111) as compared to the cubic AlN on Si (111).

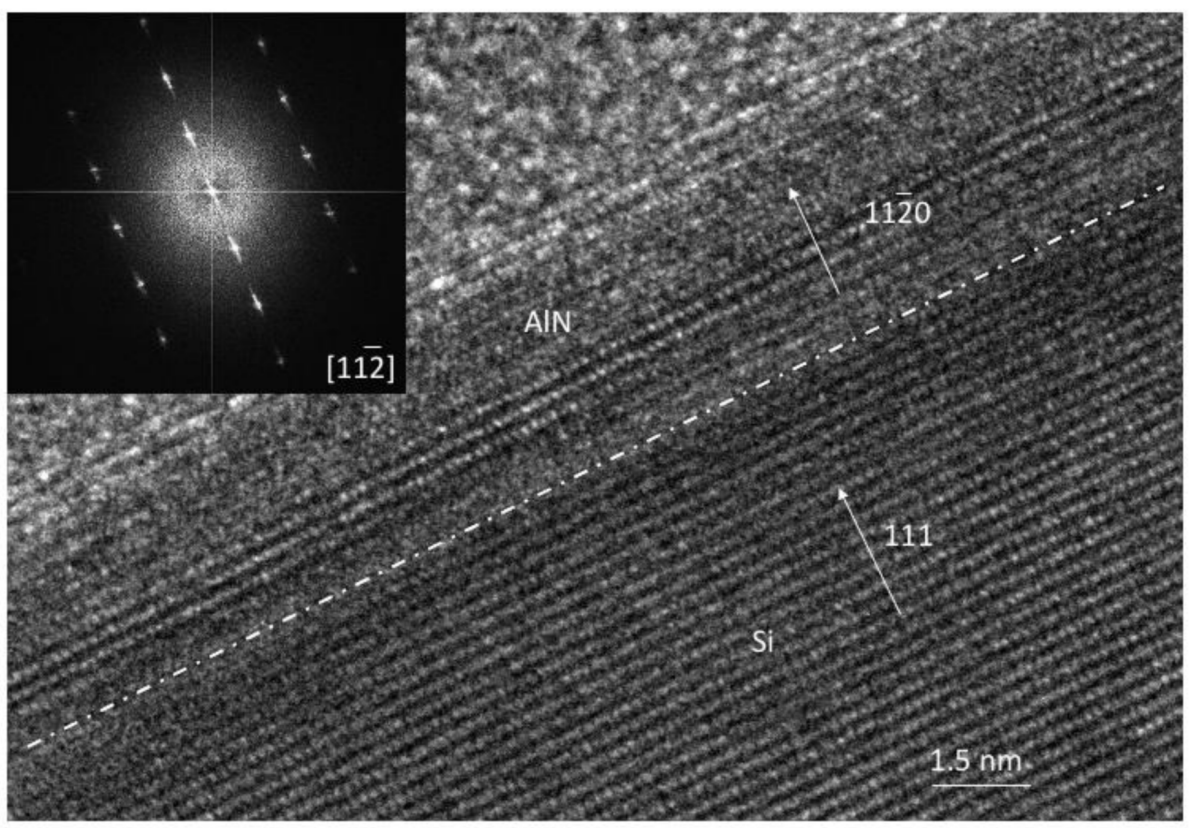

Figure 3. HRTEM image showing a rather sharp AIN/Si interface near the [11-2] zone of the substrate. FFT from the substrate and the film has been shown as an inset.

To investigate the crystal structure of the film on GaN/shappire, XRD was used. Initially, we obtain XRD on the GaN/sapphire substrate (see Figure 4). It shows clearly the (0001) oriented sapphire and wurtzitic GaN with (0002) and (0004) peaks. Figure 4 shows 
a series of XRD scans on AlN films deposited after a different number of cycles: 50, 100, 250 , and 500 cycles, respectively, at $380^{\circ} \mathrm{C}$. A broad peak was observed on the right side of the (0002) GaN diffraction peak. The intensity of this broad peak appeared to increase with the increase in number of cycles. The d-spacing of the broad peak is $0.251 \mathrm{~nm}$, which conforms the d-spacing of 111 zinc blend (cubic) AlN. We observe that the film quality degrades when the deposition temperature is lower. We have employed here grazing incidence small angle X-ray scattering to obtain the optimum growth temperature effects. Our results show that the optimum growth temperature is $420^{\circ} \mathrm{C}$. The increase in growth temperature gives better film; in particular, it lowers the impurity content in the film. The objective here is to grow epitaxial AlN at a lowest possible temperature. We observe that the film is not epitaxial at a temperature of $360^{\circ} \mathrm{C}$ in the series [32]. The temperature effects on crystal structure and the transformation kinetics are beyond the scope of the present work. In addition, the grazing incidence small angle $\mathrm{X}$-ray scattering has been used to study the morphology of the surface during AlN growth on (11-20) sapphire by atomic layer deposition [32]. They reported that the films are textured aluminum nitride, and XRD scans show a (1010) peak, indicating wurtzitic (hexagonal) AlN. Note that in the present work, the orientation of sapphire is (0001) and the AlN was deposited on (0001) GaN. This suggests that the orientation of the layer on which it is deposited plays a major role in detecting the crystal structure of AlN.

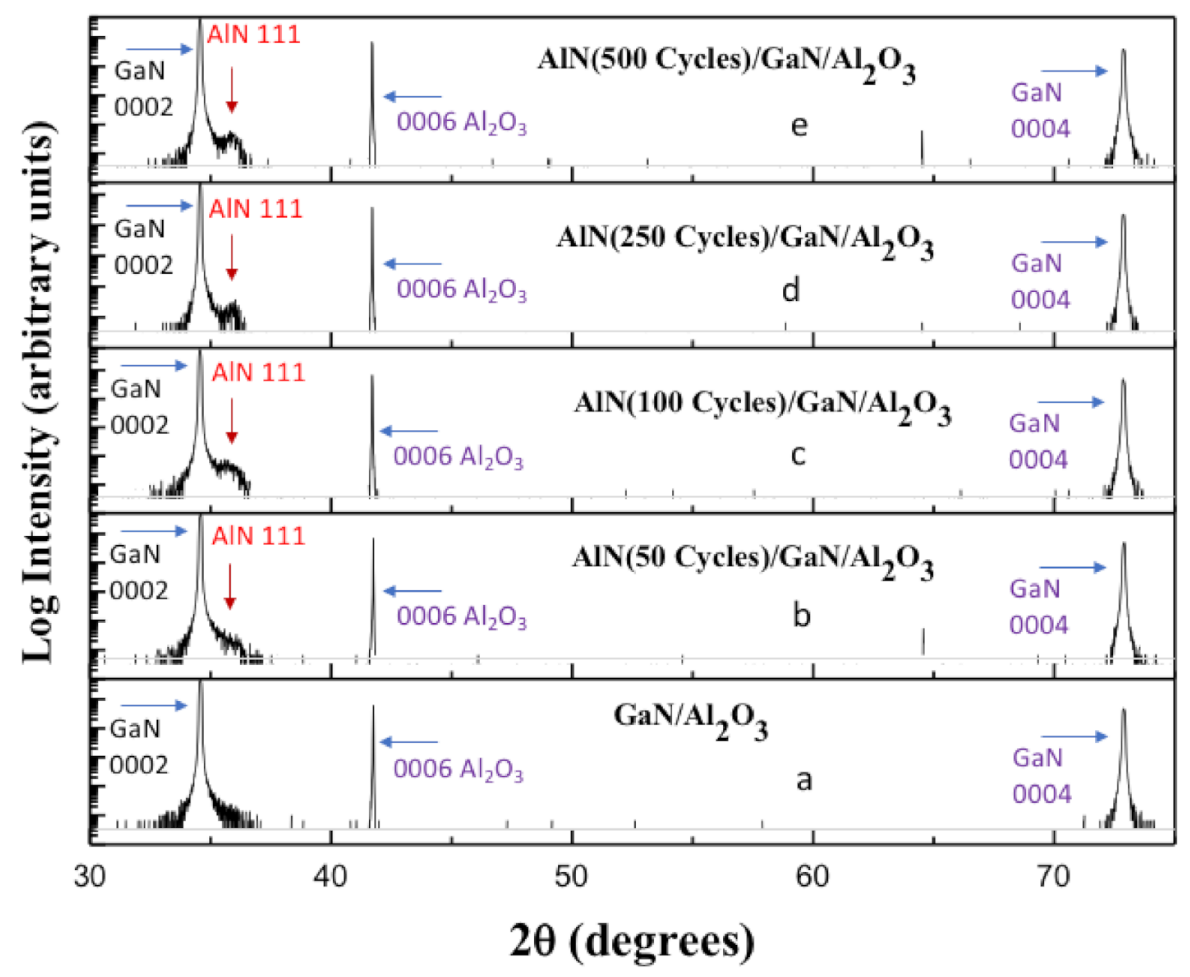

Figure 4. A series of XRD patterns in log scale from AlN films on GaN/sapphire as a function of number of reaction cycles at $420^{\circ} \mathrm{C}$. XRD on GaN/sapphire is shown at the bottom panel.

TEM was carried out on these films to further investigate the interfaces and structural characteristics of the AlN films deposited on GaN/sapphire substrate templates. We at first investigate the GaN/sapphire interface. Figure 5a is an HRTEM image showing the $\mathrm{GaN}$ on (0001)-oriented sapphire. Threading dislocations were observed to nucleate at the interface. It shows that the (0001) plane of GaN is parallel to the (0001) plane of sapphire. A low-magnification TEM showing GaN and sapphire layers is given as an inset. Figure $5 b$ is the high-magnification HRTEM image of GaN close to the [1-210] zone showing the 0001 $(\mathrm{d}$-spacing $=0.51 \mathrm{~nm})$ and 10-10 lattice fringes. In this zone, the (0001) plane appears as a result of double diffraction. The corresponding fast Fourier transform (FFT) shows the 
0001, 0002, 10-10, and 10-11 reflections of wurzitic GaN close to the [1-210] zone (see the index of Figure 5b), which were obtained from the HRTEM image.
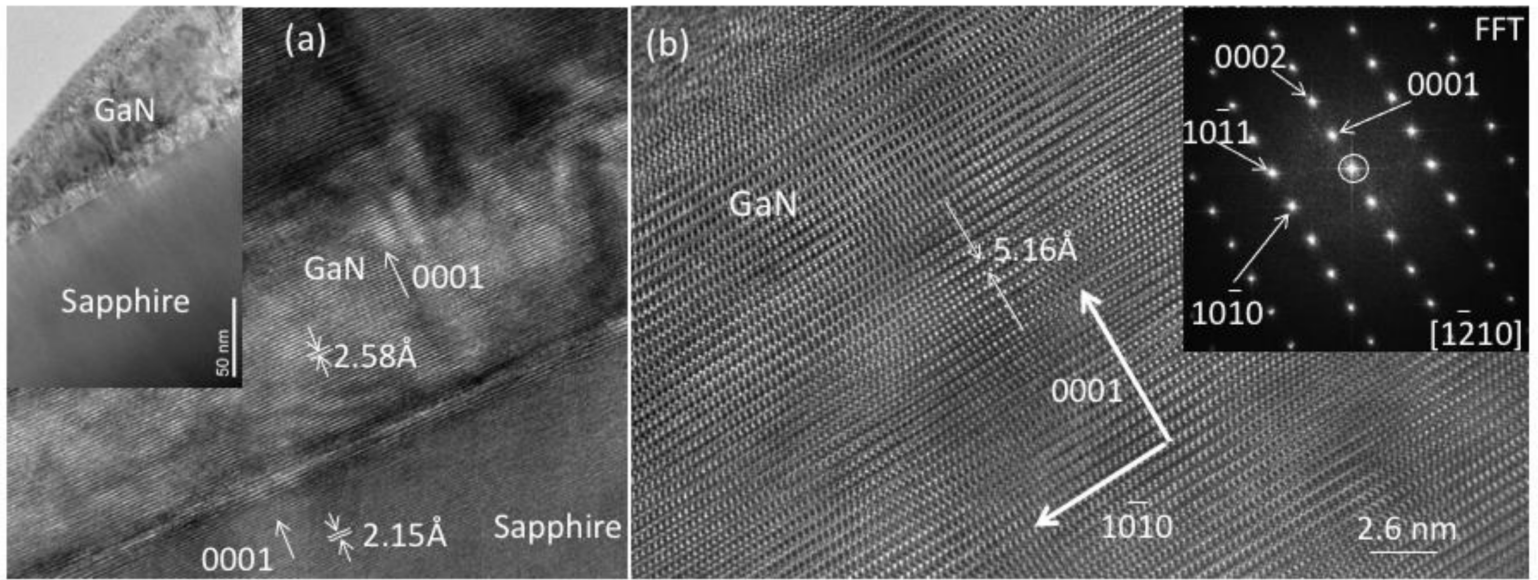

Figure 5. (a) TEM image showing GaN film on (0001) sapphire. (b) HRTEM image showing the lattice (0001) and (10-10) planes close to the [1-210] zone. The FFT from the HRTEM image is shown as an inset.

The AlN film, grown on (0001) GaN, exhibited an orientation relationship, see Figure 6a-e, that can be written as $(0001)_{\mathrm{GaN}}||\{111\}_{\mathrm{AlN}}$ and $\left.\left.<1210\right\rangle_{\mathrm{GaN}}||<220\right\rangle_{\mathrm{AlN}}$. Figure $6 \mathrm{~b}, \mathrm{c}$ are the FFTs obtained from AlN and GaN layers, respectively. The FFT from the AlN appears complex. The bright spots were indexed as the fcc (zinc blend) AlN close to [110] zone (see Figure $6 \mathrm{~d}, \mathrm{e})$. In this zone, two sets of $\{111\}$ planes with spacing $0.251 \mathrm{~nm}$ were observed. The angle between 111 spots is $70.5^{\circ}$, suggesting it is indeed a cubic crystal. The extra spots in Figure $6 \mathrm{~b}, \mathrm{~d}$ appear most probably due to intermixing during the growth process. Note that the growth process uses the metal-organic precursors and reactive radicals from a plasma source. The intermixing reaction layer, somewhat disordered, is approximately 8 to $10 \mathrm{~nm}$ thick (see Figure 7). This layer consists of two phases, cubic AlN and possibly wurtzitic AlGaN. As we go away from the GaN, the fraction of AlN increases. Using density functional theory and the quasi-harmonic approximation to study the phase diagram of AlN, it was shown by Seigel et al. [33] that there is a small energy difference between the wurtzite and zinc blende phases. Due to this small difference in energy, the zinc-blende phase of AlN could be stabilized due to the strain created by the substrate.
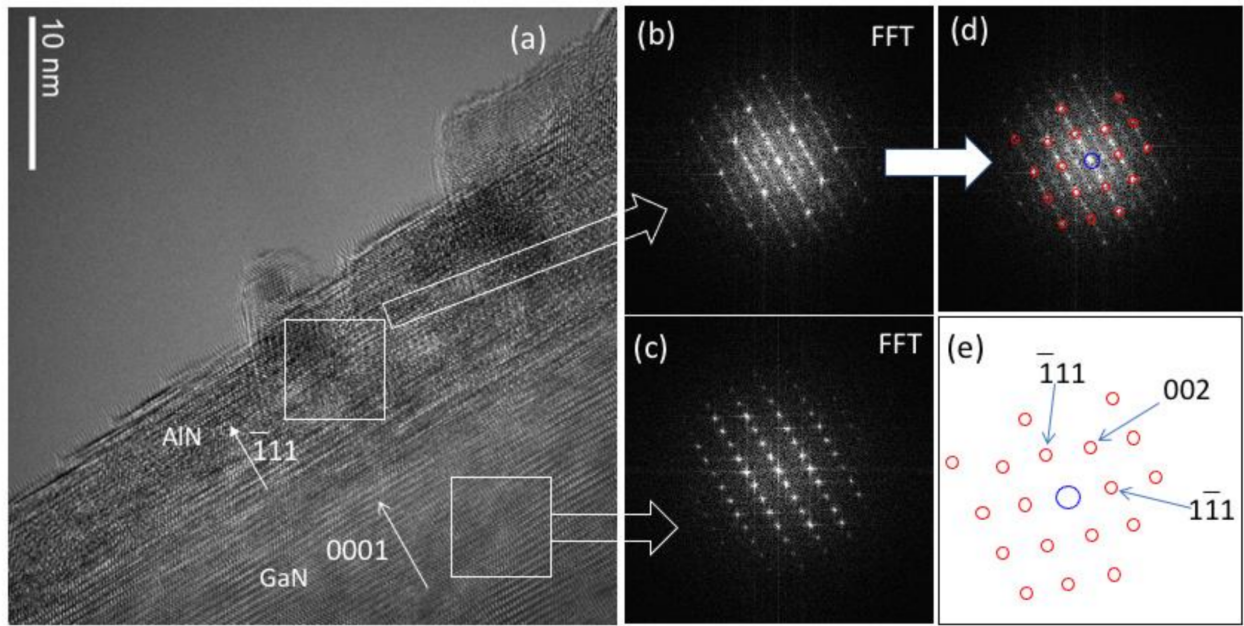

Figure 6. (a) HRTEM image showing the AlN layer grown on (0001) GaN. (b) FFT from AlN layer, (c) FFT from GaN, (d) FFT showing the encircled spots in [110] zone of zinc blend AlN, (e) The indexed pattern in [110] zone. 


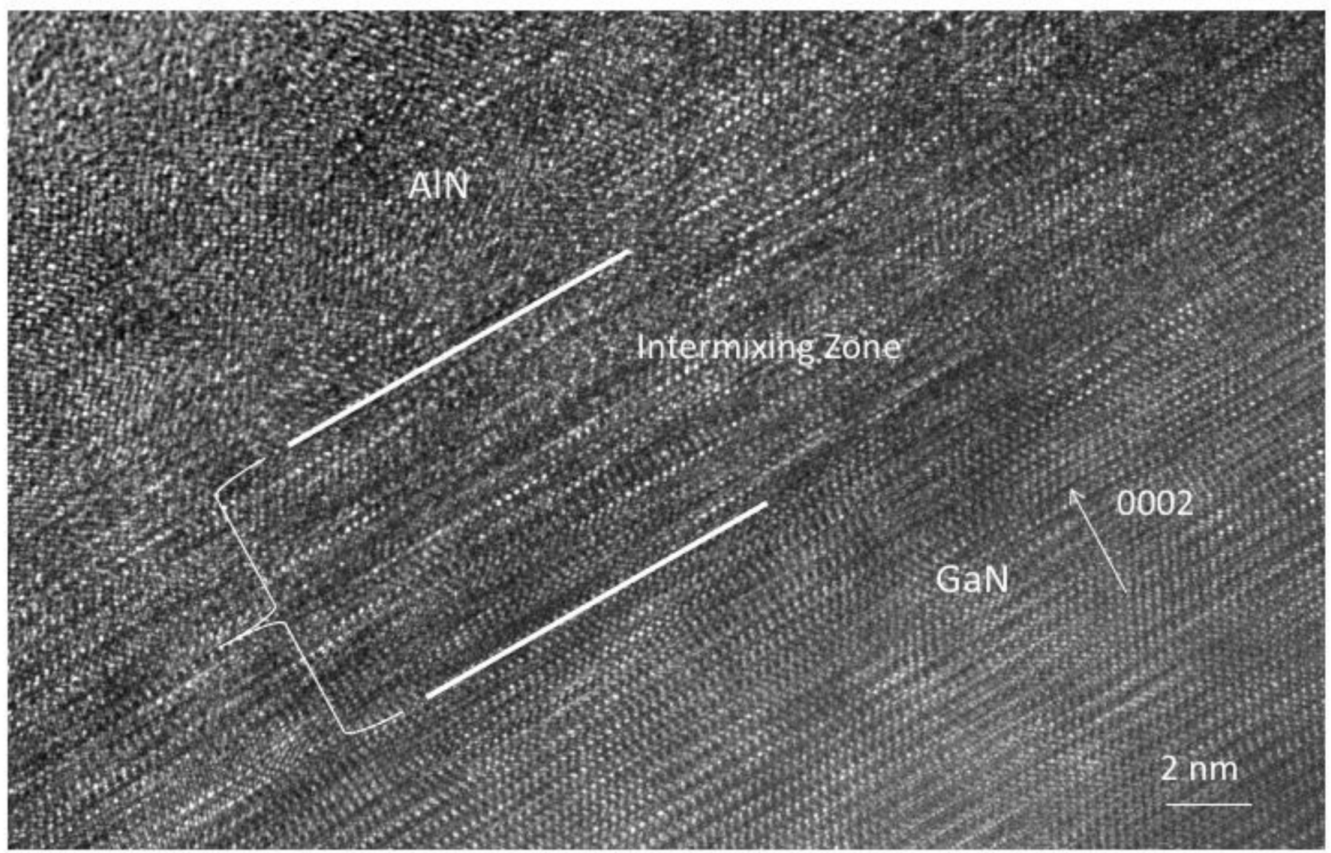

Figure 7. HRTEM showing the intermixing at the AlN/GaN interface. The interface layer is approximately 6 to $8 \mathrm{~nm}$ thick.

We have estimated the strain in the observed relationship between hexagonal GaN and cubic AlN perpendicular to the growth direction. In the perpendicular direction, the (11-20) of GaN is parallel to (220) AlN. The strain, $\left(\mathrm{d}_{11-20} \mathrm{GaN}_{-} \mathrm{d}_{220}{ }^{\mathrm{AlN}}\right) / \mathrm{d}_{11-20} \mathrm{GaN}$, estimated using the d-spacings of 11-20 of GaN and 220 of AlN, turns out to be $3 \%$. Assuming that the crystal structure of AlN is hexagonal on hexagonal GaN and the (11-20) GaN is parallel to (11-20) of AlN, one could estimate the strain. The estimated strain in this case would be $5 \%$ perpendicular to the growth direction. This suggests that the strain would be substantially lowered for cubic-AlN on hexagonal GaN as compared to the hexagonal AlN on hexagonal GaN. In particular, the metastable cubic phase can be stabilized close to the substrate. Note also that we are growing the layer at non-equilibrium conditions, which could induce metastable phases. As you go away from the substrate, the strain effect would be substantially reduced and the stable phase of AlN, hexagonal phase, can nucleate. For the growth on the Si substrate, we observe the stable hexagonal phase on (111) $\mathrm{Si}$, which suggests that the $111 \mathrm{Si}$ surface promotes the hexagonal phase. All these confirm that the strain plays a greater role in dictating the crystal structure upon growth, as the energy difference between hexagonal and cubic AlN is small. More studies are required in the future to optimize the AlN growth at low temperatures, considering all variables and their interactions.

\section{Summary and Conclusions}

In conclusion, we demonstrated the growth of ultra-thin AlN films on $\mathrm{Si}$ (111) and on $\mathrm{GaN} /(0001)$ sapphire substrate using atomic layer epitaxy at relatively low temperatures. In case of the Si substrate, the AlN film is epitaxial on (111) Si with no threading dislocations. It has deposited with a hexagonal structure with a sharp interface with Si. Similarly, the XRD results showed that the AlN is grown epitaxially with cubic phase on the GaN/(0001) sapphire substrate, which is consistent with the TEM studies. Furthermore, TEM studies showed that the interface is not sharp, containing a transition layer with two phases GaAlN and AlN. The deposited AlN film is stabilized in metastable cubic phase close to the interface. We showed that the nature of the substrate and the resulting the strain at the interface play a major role in dictating the crystal structure of AlN. 
Author Contributions: Conceptualization, R.G. and C.E.J.; methodology, N.N. and C.E.J.; formal analysis, R.G. and S.Q.; writing-original draft preparation, R.G.; writing-review and editing, C.E.J. and S.Q.; funding acquisition, C.E.J. All authors have read and agreed to the published version of the manuscript.

Funding: U.S. Naval Research Laboratory funded this work (the 6.1 research program).

Institutional Review Board Statement: Not Applicable.

Informed Consent Statement: Not Applicable.

Data Availability Statement: Data is contained within the article

Acknowledgments: We like to thank the grand challenge program on Ultrathin Multicomponent Electronic Materials.

Conflicts of Interest: The authors declare no conflict of interest.

\section{References}

1. Nepal, N.; Mahadik, N.A.; Nyakiti, L.O.; Qadri, S.B.; Mehl, M.J.; Hite, J.K.; Eddy, J.C.R. Epitaxial Growth of Cubic and Hexagonal InN Thin Films via Plasma-Assisted Atomic Layer Epitaxy. Cryst. Growth Des. 2013, 13, 1485-1490. [CrossRef]

2. Nepal, N.; Qadri, S.B.; Hite, J.K.; Mahadik, N.A.; Mastro, M.A.; Eddy, C.R. Epitaxial growth of AlN films via plasma-assisted atomic layer epitaxy. Appl. Phys. Lett. 2013, 103, 082110. [CrossRef]

3. Vurgaftman, I.; Meyer, J.R.; Ram-Mohan, L.R. Band parameters for III-V compound semiconductors and their alloys. J. Appl. Phys. 2001, 89, 5815-5875. [CrossRef]

4. Ho, I.; Stringfellow, G.B. Solid phase immiscibility in GaInN. Appl. Phys. Lett. 1996, 69, 2701-2703. [CrossRef]

5. Jain, S.C.; Willander, M.; Narayan, J.; Van Overstraeten, R. III-nitrides: Growth, characterization, and properties. J. Appl. Phys. 2000, 87, 965-1006. [CrossRef]

6. Bardhan, A.; Mohan, N.; Chandrasekar, H.; Ghosh, P.; Rao, D.V.S.; Raghavan, S. The role of surface roughness on dislocation bending and stress evolution in low mobility AlGaN films during growth. J. Appl. Phys. 2018, 123, 165108. [CrossRef]

7. Raghavan, S. Kinetic approach to dislocation bending in low-mobility films. Phys. Rev. B 2011, 83, 052102. [CrossRef]

8. Hirayama, H.; Fujikawa, S.; Norimatsu, J.; Takano, T.; Tsubaki, K.; Kamata, N. Fabrication of a low threading dislocation density ELO-AlN template for application to deep-UV LEDs. Phys. Status Solidi C 2009, 6, S356-S359. [CrossRef]

9. Chen, Z.; Fareed, R.S.Q.; Gaevski, M.; Adivarahan, V.; Yang, J.W.; Khan, A.; Mei, J.; Ponce, F.A. Pulsed lateral epitaxial overgrowth of aluminum nitride on sapphire substrates. Appl. Phys. Lett. 2006, 89, 081905. [CrossRef]

10. Zeimer, U.; Kueller, V.; Knauer, A.; Mogilatenko, A.; Weyers, M.; Kneissl, M. High quality AlGaN grown on ELO AlN/sapphire templates. J. Cryst. Growth 2013, 377, 32-36. [CrossRef]

11. Imura, M.; Nakano, K.; Narita, G.; Fujimoto, N.; Okada, N.; Balakrishnan, K.; Iwaya, M.; Kamiyama, S.; Amano, H.; Akasaki, I.; et al. Epitaxial lateral overgrowth of AlN on trench-patterned AlN layers. J. Cryst. Growth 2007, 298, 257-260. [CrossRef]

12. Paduano, Q.; Weyburne, D. Optimized Coalescence Method for the Metalorganic Chemical Vapor Deposition (MOCVD) Growth of High Quality Al-Polarity AlN Films on Sapphire. Jpn. J. Appl. Phys. 2005, 44, L150-L152. [CrossRef]

13. Sang, L.W.; Qin, Z.X.; Fang, H.; Dai, T.; Yang, Z.J.; Shen, B.; Zhang, G.Y.; Zhang, X.P.; Xu, J.; Yu, D.P. Reduction in threading dislocation densities in AlN epilayer by introducing a pulsed atomic-layer epitaxial buffer layer. Appl. Phys. Lett. 2008, 93, 122104. [CrossRef]

14. Banal, R.G.; Funato, M.; Kawakami, Y. Initial nucleation of AlN grown directly on sapphire substrates by metal-organic vapor phase epitaxy. Appl. Phys. Lett. 2008, 92, 241905. [CrossRef]

15. Banal, R.G.; Funato, M.; Kawakami, Y. Surface diffusion during metalorganic vapor phase epitaxy of AlN. Phys. Status Solidi C 2009, 6, 599-602. [CrossRef]

16. Chen, Z.; Newman, S.; Brown, D.; Chung, R.; Keller, S.; Mishra, U.K.; DenBaars, S.P.; Nakamura, S. High quality AlN grown on $\mathrm{SiC}$ by metal organic chemical vapor deposition. Appl. Phys. Lett. 2008, 93, 191906. [CrossRef]

17. Miyake, H.; Nishio, G.; Suzuki, S.; Hiramatsu, K.; Fukuyama, H.; Kaur, J.; Kuwano, N. Annealing of an AlN buffer layer in N2-CO for growth of a high-quality AIN film on sapphire. Appl. Phys. Express 2016, 9, 025501. [CrossRef]

18. Miyake, H.; Lin, C.-H.; Tokoro, K.; Hiramatsu, K. Preparation of high-quality AlN on sapphire by high-temperature face-to-face annealing. J. Cryst. Growth 2016, 456, 155-159. [CrossRef]

19. Yoshizawa, R.; Miyake, H.; Hiramatsu, K. Effect of thermal annealing on AlN films grown on sputtered AlN templates by metalorganic vapor phase epitaxy. Jpn. J. Appl. Phys. 2018, 57, 01AD05. [CrossRef]

20. Hearne, S.; Chason, E.; Han, J.; Floro, J.A.; Figiel, J.; Hunter, J.; Amano, H.; Tsong, I.S.T. Stress evolution during metalorganic chemical vapor deposition of GaN. Appl. Phys. Lett. 1999, 74, 356-358. [CrossRef]

21. Zhu, M.; Zhang, X.; Wang, S.; Yang, H.; Cui, Y. Epitaxial growth and optical characterization of AlInGaN quaternary alloys with high $\mathrm{Al} /$ In mole ratio. J. Mater. Sci. Mater. Electron. 2015, 26, 705-710. [CrossRef] 
22. Takagi, S.; Kim, S.-H.; Yokoyama, M.; Zhang, R.; Taoka, N.; Urabe, Y.; Yasuda, T.; Yamada, H.; Ichikawa, O.; Fukuhara, N.; et al. High mobility CMOS technologies using III-V/Ge channels on Si platform. Solid State Electron. 2013, 88, 2-8. [CrossRef]

23. Zhang, J.P.; Wang, H.M.; Sun, W.H.; Adivarahan, V.; Wu, S.; Chitnis, A.; Chen, C.Q.; Shatalov, M.; Kuokstis, E.; Yang, J.W.; et al. High-quality AlGaN layers over pulsed atomic-layer epitaxially grown AlN templates for deep ultraviolet light-emitting diodes. J. Electron. Mater. 2003, 32, 364-370. [CrossRef]

24. Kakanakova-Georgieva, A.; Nilsson, D.; Janzén, E. High-quality AlN layers grown by hot-wall MOCVD at reduced temperatures. J. Cryst. Growth 2012, 338, 52-56. [CrossRef]

25. Uehara, K.; Aota, Y.; Kameda, S.; Nakase, H.; Isota, Y.; Tsubouchi, K. Growth of Atomically Flat-Surface Aluminum Nitride Epitaxial Film by Metalorganic Chemical Vapor Deposition. Jpn. J. Appl. Phys. 2005, 44, 2987-2992. [CrossRef]

26. Hanlon, A.; Pattison, P.M.; Kaeding, J.F.; Sharma, R.; Fini, P.; Nakamura, S. 292 nm AlGaN Single-Quantum Well Light Emitting Diodes Grown on Transparent AlN Base. Jpn. J. Appl. Phys. 2003, 42, L628-L630. [CrossRef]

27. Lobanova, A.; Yakovlev, E.; Talalaev, R.; Thapa, S.; Scholz, F. Growth conditions and surface morphology of AlN MOVPE. J. Cryst. Growth 2008, 310, 4935-4938. [CrossRef]

28. Balaji, M.; Claudel, A.; Fellmann, V.; Gelard, I.; Blanquet, E.; Boichot, R.; Pierret, A.; Attal-Tretout, B.; Crisci, A.; Coindeau, S.; et al. Effects of AlN nucleation layers on the growth of AlN films using high temperature hydride vapor phase epitaxy. J. Alloys Compd. 2012, 526, 103-109. [CrossRef]

29. Abdulagatov, A.I.; Ramazanov, S.M.; Dallaev, R.S.; Murliev, E.K.; Palchaev, D.K.; Rabadanov, M.K.; Abdulagatov, I.M. Atomic Layer Deposition of Aluminum Nitride Using Tris(diethylamido)aluminum and Hydrazine or Ammonia. Russ. Microelectron. 2018, 47, 118-130. [CrossRef]

30. Jung, Y.C.; Hwang, S.M.; Le, D.N.; Kondusamy, A.L.N.; Mohan, J.; Kim, S.W.; Kim, J.H.; Lucero, A.T.; Ravichandran, A.; Kim, H.S.; et al. Low Temperature Thermal Atomic Layer Deposition of Aluminum Nitride Using Hydrazine as the Nitrogen Source. Materials 2020, 13, 3387. [CrossRef]

31. Knoops, H.C.M.; Faraz, T.; Arts, K.; Kessels, W.M.M. (Erwin) Status and prospects of plasma-assisted atomic layer deposition. J. Vac. Sci. Technol. A 2019, 37, 030902. [CrossRef]

32. Anderson, V.R.; Nepal, N.; Johnson, S.D.; Robinson, Z.R.; Nath, A.; Kozen, A.C.; Qadri, S.B.; Demasi, A.; Hite, J.K.; Ludwig, K.F., Jr.; et al. Plasma-assisted atomic layer epitaxial growth of aluminum nitride studied with real time grazing angle small angle x-ray scattering. J. Vac. Sci. Technol. A 2017, 35, 031508. [CrossRef]

33. Siegel, A.; Parlinski, K.; Wdowik, U.D. Ab initiocalculation of structural phase transitions in AlN crystal. Phys. Rev. B 2006, 74. [CrossRef] 